\title{
Research and Development of Calibration Device on SF_6 Gas Density Monitoring Equipment
}

\author{
Hu Chengbo ${ }^{1, \text { a }}$, Ma Yong ${ }^{2, \mathrm{~b}}$, Zhou Zhicheng ${ }^{3, \mathrm{c}}$, Tao Fengbo ${ }^{4, \mathrm{~d}}$, Xu Changfu $^{5, \mathrm{e}}$ \\ 1,2,3,4,5 Jiangsu Electric Power Company Research Institute, Nanjing, 211103, China \\ aemail:69972006@qq.com, bemail:my1313@163.com, 'email:zzc0708@163.com, \\ demail:t123456@163.com, eemail:xu523@163.com,
}

Keywords: SF6 gas, cylinder piston, on-line monitoring device, calibration

\begin{abstract}
The calibration of SF6 gas density on-line monitoring device needs to simulate different pressure environment to calibrate the data accuracy of the on-line monitoring device. At present, the simulation of different pressure environment is conducted by artificial cranking bar to change the stroke of cylinder piston to realize the aim of change the gas pressure. Generally, technical personnel take calibration device to the field, select a suitable pressure, connect the checked device to test button and conduct the contrast calibration. Based on above operation, multi-group data of the checked on-line monitoring device can be obtained. While this method depends on artificial manual operation, squander time and vigour and can not set pressure value exactly. In order to make up for deficiency of existing problems, the paper presents a calibration device on SF_6 gas density monitoring equipment. The calibration device can make the calibration procession conducted automatically through controlling the pressure and its lasting time automatically. The use of the device can effectively complete calibration and improve the quality of work.Facts show that it can provide a powerful guarantee to grid equipment reliable operation.
\end{abstract}

\section{Introduction}

As the connection between power generation and electricity consumption, high voltage circuit breaker operates reliably is of signality in guaranteeing grid safety. In recent ten years, the average loss of electric quantity in every circuit breaker accident reached millions kwh. The losses it leaded is several thousand fold of the equipment value. Therefore, power operation departments put forward impending need for the reliable operation of high voltage circuit breaker. Because of the excellent arc extinguishing resistance, insulating properties, reliable chemical and avirulence , SF6 gas is used as high voltage switch, insulationand arc-suppression medium of equipment successfully since 1960s. It is also used as circuit breaker of EHV and the unique arc-suppression medium of GIS. The usage of SF6 has caused a great revolution of high voltage switch.

In order to ensure the grid equipment operate safe and reliable, grid departments conduct preventive test to electric equipment every year and maintain equipment according to the test result. Circuit breaker state monitoring creates condition to the transformation from scheduled maintenance to state maintenance. For a long time, scheduled maintenance and blind disintegration not only waste a large number of human resource, but also cause power loss and reduce the equipment life expectancy[1-2].

With the development of computer technology, signal processing, artificial intelligence and sensing technology, electric equipment running state can be achieved on-line monitoring. This method can find equipment defects in time, degrade the incidence of accident, reduce frequency of preventive test and workload of maintenance. Accuracy of on-line monitoring device will decide the accuracy of equipment state directly. Therefore, in order to ensure high voltage circuit breaker work safely, promote reliability of electric equipment operation, it is very important to calibrate the on-line monitoring device. It has significant economic value in theory and practice[3]. 


\section{Status and Problems}

The calibration of SF6 gas density on-line monitoring device needs to simulate different pressure environment to calibrate the data accuracy of the on-line monitoring device. At present, the simulation of different pressure environment is conducted by artificial cranking bar to change the stroke of cylinder piston to realize the aim of change the gas pressure. Generally, technical personnel take calibration device to the field, select a suitable pressure, connect the checked device to test button and conduct the contrast calibration. Based on above operation, multi-group data of the checked on-line monitoring device can be obtained.

Disadvantages of the existing calibration method is obvious.

(1)Calibration needs artificial cranking bar to change the stroke of cylinder piston to realize the aim of change the gas pressure. Replicate tests waste a large number of human resource and lead to low working efficiency.

(2)Without automatic controlling function the whole process depends on manual operation. It is unable to set the pressure value precisely.

(3)The record and contrast of calibration data relay on artificial manipulation, it can easily cause artificial errors.

(4)Calibration results have not realize on-line maintenance. It is inconvenient to establish on-line monitoring device calibration record and afterwards retrieval.

(5)The development of calibration work needs experienced professional personnel. With the popular of transformation on-line monitoring device, the maintenance should be conducted regularly. It will cost a large number of human resources[4].

\section{Technical program}

In order to solve the above problems, this paper presents a new device for intelligent check of SF6 gas density on-line monitoring equipment. It can realize control pressure value and lasting time automatically and make the calibration work conducted automatically.

To solve the problems, this paper presents such a technical program.

The calibration device on SF6 gas density monitoring equipment includes storage cylinder, variable cylinder and the control unit. The storage cylinder connects to the variable cylinder through NO.1 electromagnetic valve. There are sensors between NO.1 electromagnetic valve and the variable cylinder. On the variable cylinder, there is a test interface. This interface connects to the variable cylinder by NO.2 electromagnetic valve. The control unit connects to NO.1 electromagnetic valve, NO.2 electromagnetic valve and variable cylinder.

The variable cylinder includes electric putter. The electric putter connects to the piston of variable cylinder and it is controlled by control unit. The sensors include temperature sensor and pressure sensor.

The device has such advantages. By means of control unit, NO.1 electromagnetic valve, NO.2 electromagnetic valve and variable cylinder can control pressure value and its lasting time to calibrate the equipment automatically. This method can save a lot of time and human resource[5].

\section{Calibration steps}

The calibration device on SF6 gas density monitoring equipment includes storage cylinder 1, variable cylinder 2 and the control unit 3. The storage cylinder 1 connects to the variable cylinder 2 through NO.1 electromagnetic valve 4. There are sensors between NO.1 electromagnetic valve 4 and the variable cylinder 2 . On the variable cylinder 2 , there is a test interface 5 . This interface 5 connects to the variable cylinder 2 by NO.2 electromagnetic valve 6 . The control unit 3 connects to NO.1 electromagnetic valve 4, NO.2 electromagnetic valve 6 and variable cylinder 2 . The device diagram as is shown in figure 1.

The variable cylinder 2 includes electric putter 21 . The electric putter 21 connects to the piston 
22 of variable cylinder 2 and it is controlled by control unit 3. The sensors include temperature sensor 7 and pressure sensor 8.

Before calibration, NO.1 electromagnetic valve 4 and NO.2 electromagnetic valve 6 remain closed. Set pressure value of test interface 5 through control unit 3. According to the demand of calibration, technical personnel can set several values of pressure and their lasting time. Control unit 3 opens NO.1 electromagnetic valve 4 and control the electric putter 21 retreat to make the pressure value of variable cylinder 2 approach to the preset value. Then, close NO.1 electromagnetic valve 4 . Connect the checked SF6 gas density on-line monitoring equipment to test interface 5 . The control unit 3 opens NO.2 electromagnetic valve 6 to make the pressure value of test interface 5 is equal to the pressure value of variable cylinder 2 . According to the pressure signals from pressure sensor 8 , control unit 3 control electric putter 21. When the pressure value of variable cylinder 2 approach is equal to the preset value, start calibrating.

On the basis of the preset order and time, when the calibration finished, control unit should open NO.1 electromagnetic valve 4 and control electric putter 21 to go ahead to press the gas into storage cylinder 1 . The work flow as is shown in figure 2.

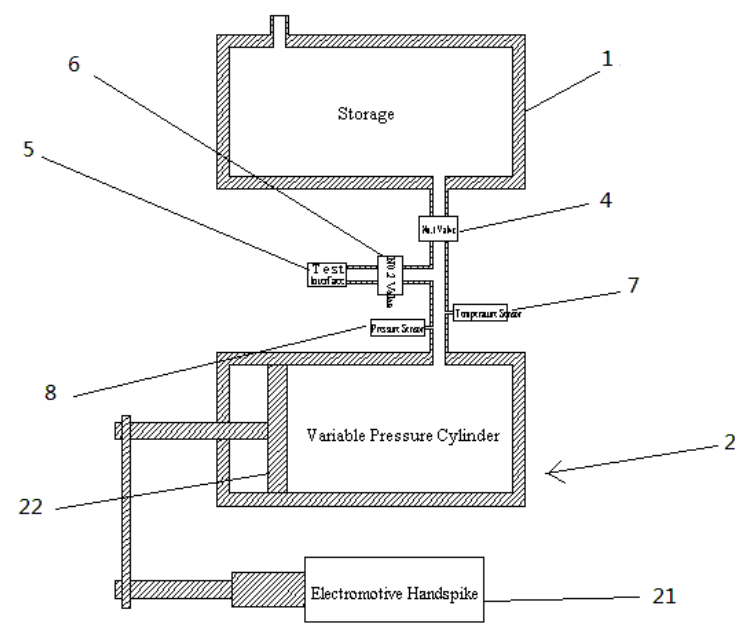

Fig 1: The device diagram

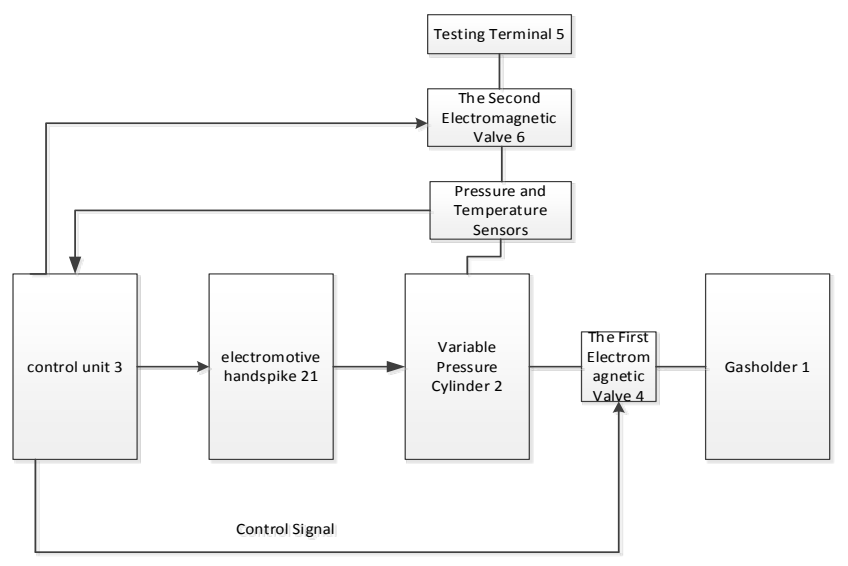

Fig 2: The calibration work flow

\section{Function description}

SF6 gas density standard signal source simulates the gas pressure of SF6 air chamber. The output gas pressure will be changed automatically by motor. It will be used as the calibration source.

(1)Adopt double cylinders model. One is used as storage cylinder to store SF6 gas as the source, the other is used as variable cylinder to provide output signal.

(2)The volume of variable cylinder can be changed through electric putter driving piston of cylinder.

(3)Adopt electromagnetic valve to control pipeline make-break.

(4)Adopt HALL sensor and infrared sensor to guarantee the electric putter operates in a safety range.

(5)Adopt temperature sensor to detect internal temperature of cylinder. Adopt pressure sensor to detect internal pressure of cylinder.

(6)Signal source can exchange data with host computer through WIFI interface.

(7)5 groups of different pressure can be prestored.

(8)Receive the 5 groups of different pressure prestored from host computer.

(9)Have the function of liquid crystal display.

\section{Design scheme}

The architecture of sample arm $11+$ fpga, 3 channels sample(individual samples adopt time 
division multiplexing to acquisite the temperature and pressure of variable cylinder ), $6 \mathrm{I} / \mathrm{O}$ output to control electromagnetic valve and electric putter.

\section{DAC circuit}

Adopt DAC7731E chip from ADI company. DAC7731E chip is 16 bit resolution DAC. It is characterized by lower exhaustiveness. Maximum power is $150 \mathrm{mw}$, internal reference voltage is $10 \mathrm{v}$.

2.Following circuit

Before pressure and temperature entering ADC, use first order following circuit to improving precision. These amplifiers are unit gain stable angina, typical gain bandwidth is $4 \mathrm{MHz}$.

3.Fpga chip type

Adopt EP3C5E144C8N from ALTERA. LAC/CLB number:321. Logical element/unit number: 5136. RAM bit number: 423649. Input/output number: 94.

\section{4.arm11}

Adopt arm11 can operate linux perfectly and realize more intelligent program design.

\section{Temperature sensor}

The resistance value will change with the temperature change. Temperature sensor gets resistance value(voltage/current) through adding a exciting current and measuring voltage value. Then ,transform resistance value to temperature and measure the temperature.

There are 3 connecting ways between thermal resistance and sensor: second-line, three-line and four-line. The paper adopts three-line:connect a lead wire in the bottom of thermal resistance, on the other end connect two lead wires. This method is always compound used with electric bridge and it can eliminate influence of lead wire resistance very well.

\section{Pressure sensor}

Adopt pressure sensor from MICAUD. Precision is $0.5 \%$. Output voltage is $0-5 \mathrm{v}$. Measuring range is $01-1 \mathrm{mpa}$.

7.Infrared ranging sensor

Adopt infrared ranging sensor GP2Y0E03 from SHARP. Measuring range is $4-50 \mathrm{~cm}$. It can be used to detect process data of cylinder piston. According to the result, program can preset the aim pressure of piston and judge whether the process exceed the range.

8.Power supply

Because the motor will cost a lot of energy under a high pressure, the paper adopts 600w switching power supply to provide energy. A 24-round 5v/5w power supplies energy to arm 11 fpga wifi and provide $10 \mathrm{v} / 3 \mathrm{w}$ current to stimulate circuit through ldo decompression. Motor driver will use the energy of 24 power source directly.

\section{Conclusion}

This method solves the questions such as artificial operation, inconvenient archiving and retrieval. It meets the need of intelligent calibration of substation on-line monitoring devices and has a very wide market.

\section{References}

[1]SHEN Zhu, MEI Zhi-gang, ZHAO Wei, et al. calibrator for electronic current transformer[J]. J Tsinghua univ(sci\&tech), 2003, (3): 314-317.

[2]CLIN Min;YANG Jinggang;JIA Yongyong;ZHAO Ke;GAO Shan;ZHANG Ziyang; Research and Application on Electric Equipment SF6 Gas Leak Detection Technology[J]. Jiangsu Electrical Engineering, 2014,04.

[3]CHEN Jiulin, XU Chencheng, WU Zaijun, DOU Xiaobo, et al. Development of Transmission Line On-line Monitoring System Based On Wireless Network[J]. Jiangsu Electrical Engineering, 2013, 32(6): 39-42 
[4]NIU Wenjun,WEI Junmei,ZHANG Duo,SUN Yongheng. Research on 550kV SF2/N2 Aeration Bus Insulating[J]. High voltage apparatus,2014, 33:36-39.

[5]SHU Jia, HUANG Xin-bo, ZHAO Long, et al. Design of SF6 Gas Intelligence Monitoring Device for Smart Substation[J]. High Voltage Engineering, 2013, 49(4):1-6. 